\title{
Success rate of grafts with multiple renal vessels in 3,136 kidney transplants
}

\author{
Emre Karakaya, Aydincan Akdur, Ebru Ayvazoglu Soy, Gokhan Moray, Mehmet Haberal
}

Department of General Surgery, Baskent University Hospital, Ankara, Turkey

Background: Multiple renal vessels are often detected in living and deceased organ donors. In the past, transplant with multiple renal vessel grafts has been a contraindication because of high vascular and urological complication rates. However, improvements in vascular reconstruction and anastomosis techniques have allowed graft function to be maintained for many years. Here, we retrospectively evaluated transplant of multiple renal vessel grafts and graft survival and postoperative vascular and urological complications.

Methods: From November 1975 to July 2020, there were 3,136 renal transplants (716 deceased donors and 2,420 living donors) performed in our center. There were 2,167 living donors and 643 deceased donors with single renal vessel grafts and 253 living donors and 73 deceased donors with multiple renal vessel grafts. For anastomoses, external iliac, internal iliac, common iliac, and inferior epigastric arteries, and external iliac veins were used. Cold ischemia time, anastomosis time, postoperative vascular and urological complications, acute tubular necrosis, creatinine clearance, serum creatinine levels, graft rejection episodes, and graft and patient survival rates were evaluated.

Results: With regard to creatinine clearance, cold ischemia and anastomosis time, acute tubular necrosis, rejection episodes, and 1-, 2-, and 5-year post-transplant serum creatinine levels, there were no significant differences between the groups. Graft survival rates in the single renal vessel group were $92.9 \%$ at 1 -year post-transplant and $78.3 \%$ at 5 -year post-transplant; rates in the multiple renal vessel group were $93.1 \%$ at 1 -year and $79.7 \%$ at 5 -year. The corresponding patient survival rates were $95.5 \%$ (1-year) and $92.9 \%$ (5-year) for the single renal vessel group and $96.9 \%$ (1-year) and $87.2 \%$ (5-year) for the multiple renal vessel group.

Conclusions: Improved anastomosis and reconstruction techniques have allowed the safe transplant of multiple renal vessel grafts that may remain functional for many years.

Corresponding author: Emre Karakaya

E-mail: rectorate@baskent.edu.tr

(c) The Korean Society for Transplantation

This is an Open Access article distributed under the terms of the Creative Commons Attribution Non-Commercial License (http://creativecommons.org/licenses/by-nc/4.0/) which permits unrestricted non-commercial use, distribution, and reproduction in any medium, provided the original work is properly cited. 